\title{
Signs of a 'Time Crystal' in a surprising place
}

\author{
Sean Barrett ${ }^{a}$, Jared Rovny ${ }^{a}$, and Robert Blum ${ }^{a}$ \\ ${ }^{a}$ Yale University Physics Department, 217 Prospect St., New Haven, CT, 06520- \\ 8120, USA, sean.barrett@yale.edu
}

The periodic spatial arrangement of atoms in a crystal like $\mathrm{NaCl}$ has been known for a century, thanks to the hard work of pioneering crystallographers. The concept of a "discrete time crystal" (DTC) is a much more recent development ( 6 years old). It is one example of novel phases of matter proposed to exist in driven many-body systems. Recent experiments using ion traps and diamond NV centers have found signatures of the DTC. Here, we present nuclear magnetic resonance (NMR) observations of DTC signatures in a third, strikingly different system: an ordered spatial crystal of ammonium dihydrogen phosphate (ADP). This result appears to challenge current assumptions about what conditions are needed for DTCs to form. We will discuss how earlier $\mathrm{x}$-ray and neutron diffraction studies of ADP (and related compounds) helped us to pin down the spin Hamiltonian for ADP, which is very different from those studied in all prior work. Lastly, we demonstrate a novel DTC-echo, revealing the hidden coherence produced by the DTC sequence. These NMR studies $[1,2]$ are yet another example of how crystallographic studies can continue to pay scientific dividends, many decades after the initial work.

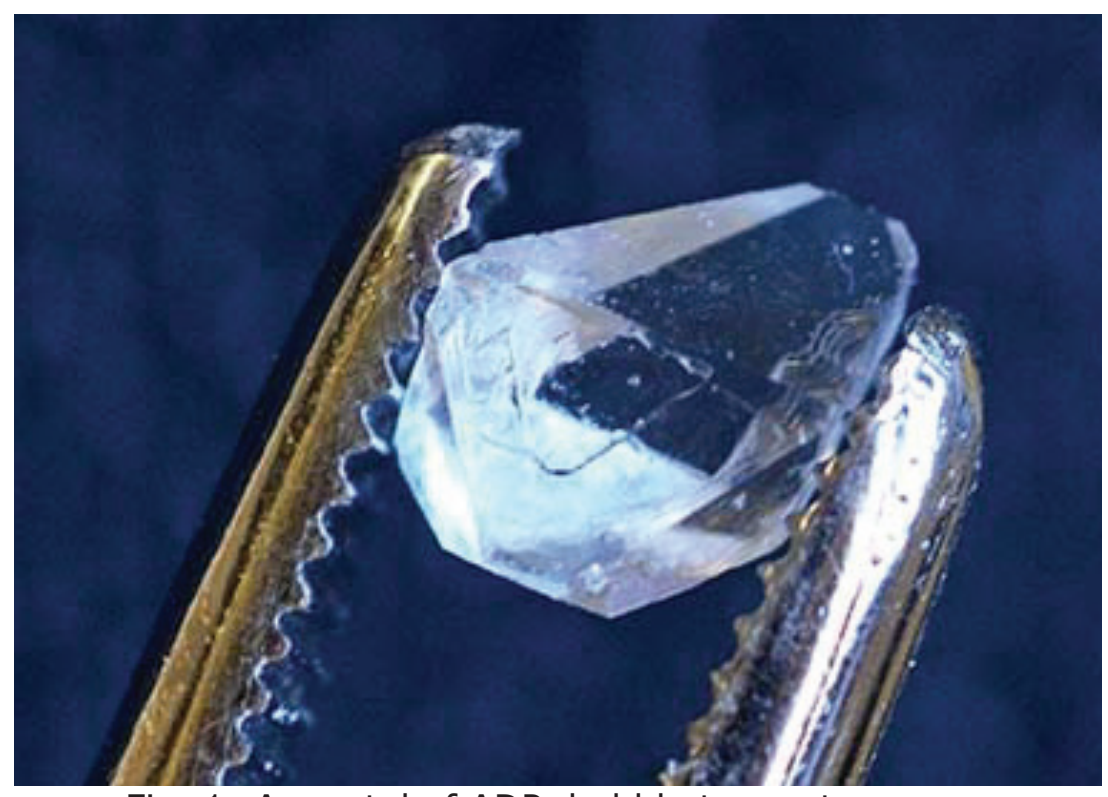

Fig. 1: A crystal of ADP, held between tweezers.

References:

[1] Rovny, J. et al. (2018). Phys. Rev. Lett. 120, 180603.

[2] Rovny, J. et al. (2018). Phys. Rev. B 97, 184301. 